\title{
ESTIMATION OF THE BINDING ABILITY OF MAIN TRANSPORT PROTEINS OF BLOOD PLASMA WITH LIVER CIRRHOSIS BY THE FLUORESCENT PROBE METHOD
}

\author{
E. A. Korolenko, ${ }^{\mathrm{a}}$ E. V. Korolik, ${ }^{\text {a* }}$ A. K. Korolik, \\ and V. V. Kirkovskii ${ }^{\mathrm{b}}$
}

UDC 535.371:616.36-004

\begin{abstract}
We present results from an investigation of the binding ability of the main transport proteins (albumin, lipoproteins, and $\alpha$-1-acid glycoprotein) of blood plasma from patients at different stages of liver cirrhosis by the fluorescent probe method. We used the hydrophobic fluorescent probes anionic 8-anilinonaphthalene-1-sulfonate, which interacts in blood plasma mainly with albumin; cationic Quinaldine red, which interacts with $\alpha-1$ acid glycoprotein; and neutral Nile red, which redistributes between lipoproteins and albumin in whole blood plasma. We show that the binding ability of albumin and $\alpha$-1-acid glycoprotein to negatively charged and positively charged hydrophobic metabolites, respectively, increases in the compensation stage of liver cirrhosis. As the pathology process deepens and transitions into the decompensation stage, the transport abilities of albumin and $\alpha$-1-acid glycoprotein decrease whereas the binding ability of lipoproteins remains high.
\end{abstract}

Key words: fluorescent probe, blood plasma, liver cirrhosis, albumin, lipoproteins, $\alpha$-1-acid glycoprotein.

Introduction. Several serious diseases, including liver cirrhosis (LC), are complicated by development in patients of endogenous intoxication syndrome that results in uncontrolled release into fluids of products of lipid and protein exchange $[1,2]$. These compounds enhance the development and progress of toxic complications that not only deepen the clinical course of the main disease but also lead directly to lethal consequences.

Many biologically active compounds, including various metabolites, toxins, and drugs, are low-molecularweight hydrophobic compounds and are transported as complexes with proteins [3, 4]. The most adequate and informative approach to diagnosing endogenous intoxication is testing of the binding ability of the main transport proteins responsible for sorption and transfer of hydrophobic metabolites and drugs. Human serum albumin (HSA), lipoproteins (LP), and $\alpha$-1-acid glycoprotein (AGP) are very important transport systems that differ not only in structure but also in degree of affinity for certain types of low-molecular-weight ligands. They are also relatively specific for binding variously charged ligands [3, 4].

The binding ability of the main transport proteins of blood plasma is currently estimated using the fluorescent probe method. It is based in principle on estimating the binding parameters of hydrophobic fluorescent probes to transport proteins. Several fluorescent probes with different charges are used in this method. The fluorescence quantum yield of them is significantly increased after binding to transport proteins and, therefore, the fluorescence of such probes is determined mainly by their bound fraction [5-8]. This enables the binding ability of transport proteins of blood plasma to hydrophobic metabolites to be estimated. The charge of the low-molecular-weight hydrophobic ligands is the main factor of protein binding. Anionic ones are primarily sorbed by HSA [9-11]; cationic, by AGP [10, 12], and uncharged ones, distributed between HSA and LP [13, 14]. Therefore, the binding of low-molecular-weight hydrophobic toxins and drugs by blood proteins can be analyzed using several fluorescent probes with different charges, e.g., anionic, 8-anilinonaphthalene-1-sulfonate (ANS); cationic, Quinaldine red (QR); and neutral, Nile red (NR).

\footnotetext{
*To whom correspondence should be addressed.
}

${ }^{\mathrm{a}}$ B. I. Stepanov Institute of Physics, National Academy of Sciences of Belarus, 68 Nezavisimosti Ave., Minsk, 220072, Belarus, e-mail: korolik@dragon.bas-net.by; ${ }^{\mathrm{b}}$ Belarussian State Medical University, Minsk. Translated from Zhurnal Prikladnoi Spektroskopii, Vol. 74, No. 4, pp. 507-511, July-August, 2007. Original article submitted April 2, 2007. 
TABLE 1. Optimal Conditions for Use of Fluorescent Probes

\begin{tabular}{|l|l|c|c|l||}
\hline \multicolumn{1}{|c|}{ Probe } & Charge & Probe conc., $\mu \mathrm{M}$ & Plasma dilution & Fluorescence recording conditions \\
\hline ANS & Anionic & 65 & $1: 100$ & $\begin{array}{l}\text { Spectrum in } 400-650 \mathrm{~nm} \\
\lambda_{\text {ex }}=370 \mathrm{~nm}, \lambda_{\max }=475 \mathrm{~nm}\end{array}$ \\
\hline QR & Cationic & 1 & $1: 15$ & $\begin{array}{l}\text { Spectrum in } 540-720 \mathrm{~nm} \\
\lambda_{\text {ex }}=496 \mathrm{~nm}, \lambda_{\max }=605 \mathrm{~nm}\end{array}$ \\
\hline NR & Uncharged & & $\begin{array}{l}\text { Synchronous scanning spectrum } \\
\lambda_{\text {ex }}=480-700 \mathrm{~nm}, \\
\lambda_{\text {rec }}=495-715 \mathrm{~nm} \\
\Delta=15 \mathrm{~nm}\end{array}$ \\
\hline
\end{tabular}

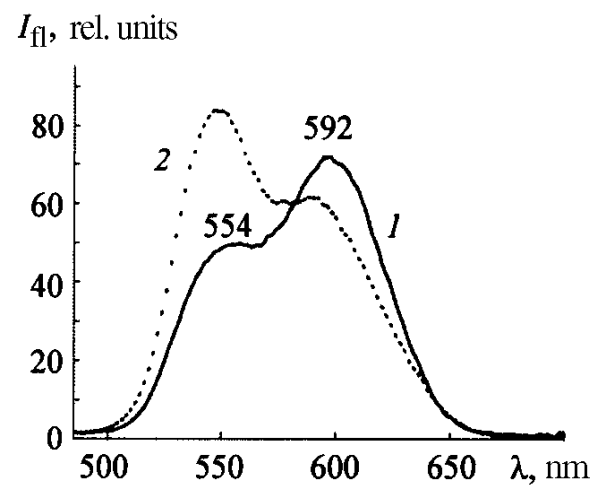

Fig. 1. Synchronous scanning spectrum of Nile red fluorescent probe in blood plasma of a healthy donor (1) and a patient with liver cirrhosis in the decompensation stage (2).

Most research on the binding ability of various proteins has until now been carried out using a very simple system of one protein and one ligand. Only scattered reports have appeared on the simultaneous use of fluorescent probes $[15,16]$ and not one on the simultaneous use of them to estimate the binding ability of plasma transport proteins with LC for diagnosis.

Our goal was to study transport features of various hydrophobic metabolites by blood plasma proteins (HSA, AGP, LP) in LC patients and to develop fluorescent tests based on them that were adapted to clinical conditions for estimating the seriousness of the pathological process.

Materials and Methods. Patients with LC (42 people) at the stage of liver-cell insufficiency were divided into three groups according to the Child-Pugh prognosis scale [17, 18]. The first group included patients with LC in the compensation stage (12 people); the second, in the subcompensation stage (12); the third, in the decompensation stage of LC (18). The diagnosis was made based on corresponding clinical, laboratory, morphological, and instrumental data. The control group was 30 healthy donors. All patients and donors gave informed consent for participation in the research.

Blood was collected from patients and donors in the morning on an empty stomach. The preservative was heparin. Blood taken from a peripheral vein was placed in a glass tube with heparin (1 mL blood to $10 \mathrm{ME}$ heparin) and centrifuged to separate plasma and cells $(2500 \mathrm{rpm}, 15 \mathrm{~min})$. Plasma was collected in plastic tubes and frozen at $-20^{\circ} \mathrm{C}$. The total protein content, total bilirubin and its fractions, moderate molecular weight molecules (data not reported), and HSA content were determined in the investigated plasma. The HSA concentration (mg/mL) was $46.8 \pm$ 2.6, $39.4 \pm 3.1,33.2 \pm 2.1$, and $24.8 \pm 1.5$ for the control, first, second, and third group, respectively. 

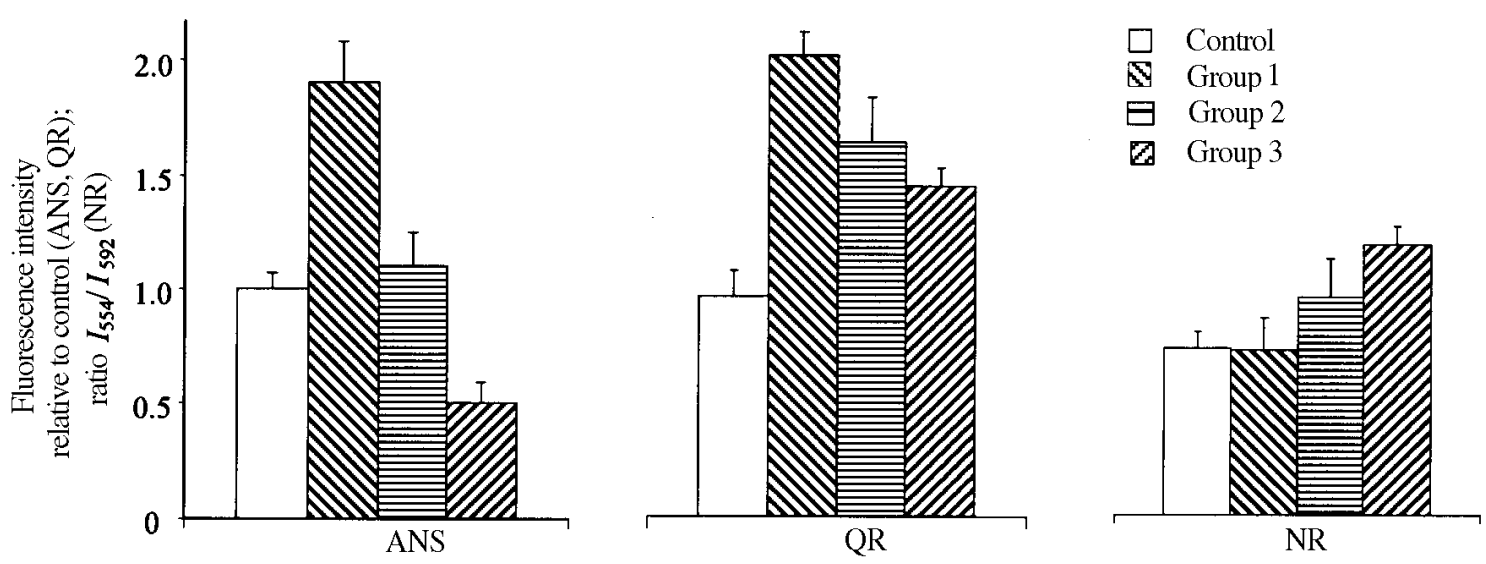

Fig. 2. Influence of liver cirrhosis on fluorescence of 8-anilinonaphthalene-1sulfonate, Quinaldine red, and Nile red in blood plasma as functions of disease stage.

We used hydrophobic fluorescent probes that were anionic, ANS (Reakhim, Moscow, Russia); cationic, QR (Reakhim, Moscow, Russia); and neutral, NR (Sigma, St. Louis, MO, USA). NR and QR probes were dissolved in ethanol. The final ethanol concentration in the investigated blood-plasma samples was less than $2 \%$. The ANS probe was dissolved in sodium phosphate buffer $(50 \mathrm{mM})$ at $\mathrm{pH} 7.4$ with $\mathrm{NaCl}(100 \mathrm{mM})$. Table 1 gives the optimal conditions for the employed probes.

Fluorescence spectra of probes were recorded on a SFL-1211A spectrofluorimeter (SOLAR, Minsk, Belarus). Fluorescence intensities of ANS, QR, and NR probes were measured at room temperature. Peak intensities of ANS, $\mathrm{QR}$, and NR probes were used to analyze the resulting data as indicators of the total binding ability of transport proteins. For the NR probe, fluorescence spectra were recorded by synchronous scanning over excitation and emission wavelengths with a constant difference between them of $\Delta \lambda=15 \mathrm{~nm}$. The ratio of fluorescence intensities of LPbound NR at $\lambda_{\max }=554 \mathrm{~nm}$ and HSA-bound NR at $\lambda_{\max }=592 \mathrm{~nm}, I_{554} / I_{592}$, was determined and reflects the distribution of NR between these two fractions in blood plasma [16] (Fig. 1).

Statistical treatment for the determination of the average value and standard deviation was performed using the standard method of Student criteria and the Microsoft Excel program.

Results. HSA is the main transport protein that binds anionic fluorescent probe ANS in blood plasma [16, 19]. The fluorescence intensity depends on both the HSA concentration in blood plasma and on its loading by metabolites and xenobiotics. At a high HSA concentration and heavy loading by hydrophobic ligands, the fluorescence intensity of ANS can be low. Therefore, we used the fluorescence intensity of ANS probe normalized to HSA concentration $\left(I_{\mathrm{ANS}} / C_{\mathrm{HSA}}\right)$ in order to obtain objective data. The fluorescence intensity of ANS that was normalized to HSA concentration and characterized the binding ability of HSA for anionic hydrophobic metabolites was much greater in blood plasma of LC patients in compensation stage than in healthy donors (Fig. 2). The binding ability of HSA (according to ANS probe) is observed to decrease for group 2 patients relative to group 1 but remains higher than in healthy donors. However, a progressive decrease of fluorescence intensity of ANS probe is seen for group 3 patients as compared with the control group and groups 1 and 2 (Fig. 2).

Neutral probe NR distributes in blood plasma between LP and HSA [16, 20, 21]. Fluorescence spectra of NR probe were obtained using the synchronous scanning method, which is widely used at present for fluorescent analysis of multi-component systems [16, 22]. The method is based on the known fact that absorption spectra maxima and fluorescence spectra of fluorophores are related and is described by the theory of the Stokes shift. For most fluorophores, the Stokes shift falls in the range 15-40 nm [23]. Using this fact, the fluorescence of multi-component systems can be described using the synchronous scanning spectrum, i.e., the simultaneous change of exciting and recording wavelengths with a constant difference between them. Such recording of the fluorescence spectrum greatly decreases the spectral overlap of bands and simplifies the analysis of multi-component mixtures without separating them. 
Figure 1 shows synchronous scanning fluorescence spectra of NR probe in blood plasma of a healthy donor and a LC patient in decompensation stage. It can be seen that the synchronous scanning spectrum of NR probe in blood plasma of both the healthy donor and the patient with decompensation LC are a broad band with maxima at 554 and $592 \mathrm{~nm}$, which correspond to LP-bound (mainly LP of low and very low density) and HSA-bound probe. Their ratio $\left(I_{554} / I_{592}\right)$ reflects the distribution of NR between these two components in blood plasma [16]. The NR probe redistributes between fractions of low and very low density LP and HSA (curve 2) in favor of LP compared to the control group (curve 1) in blood plasma of LC patients in the decompensation stage.

Analysis of NR spectra for blood plasma from LC patients indicates that $I_{554} / I_{592}$ in synchronous scanning fluorescence spectra of NR probe in blood plasma of group 1 patients does not reliably differ from the control values (Fig. 2). As the liver disease progresses (groups 2 and 3), the $I_{554} / I_{592}$ ratio increases, indicating a redistribution of NR probe binding between LP fractions of low and very low density and HSA (Fig. 2) in favor of LP.

Binding in blood plasma of cationic probe $\mathrm{QR}$, which interacts primarily with AGP $[16,24,25]$ and is monitored using the fluorescence intensity of this probe at the maximum, increases significantly for all groups of patients. This is especially evident for groups 1 and 2 (Fig. 2).

Discussion. The results indicate that the binding ability of HSA to anionic hydrophobic metabolites in LC patients at the compensation stage increases compared with the control group. The most probable reason for the increased binding ability of HSA in group 1 is the increased level of non-esterified fatty acids, the concentration of which with LC in blood plasma increases significantly [26]. The binding of high-affinity ligands, including non-esterified fatty acids, can induce conformational changes of HSA [27-29]. This in turn can create additional binding centers on this protein [28]. At the compensation stage, the contribution of LP and HSA to the transport of uncharged hydrophobic compounds (according to NR probe) does not substantially change relative to healthy donors. The binding ability of AGP for cationic hydrophobic metabolites increases sharply (according to QR probe). Because AGP is a protein of the acute phase of the inflammation and has one broad and flexible binding site [24, 25], the changes in the fluorescence intensity of QR probe are apparently due to an increased concentration of this protein in blood plasma.

For LC at the subcompensation stage, the binding ability of HSA for anionic hydrophobic ligands decreases relative to the compensation group. However, it remains greater than for healthy donors. The pathologic process for this phase typically has an increased contribution from LP and a reduced one from HSA for transport of uncharged hydrophobic metabolites (Fig. 2). These changes are probably due to a significant destruction of the protein-synthesis function of the liver and a subsequent reduction in the HSA concentration in blood plasma of patients in addition to loading of HSA by pathological metabolism products. On the other hand, liver disease is accompanied by significant destruction of lipid exchange, which leads to an increased level of LP of low and very low density in blood plasma and a reduction of the high-density LP level [30]. We found that the binding ability of AGP decreases relative to the corresponding indicator in the compensation stage. However, it remains higher than for healthy donors. This may indicate that the increased production of cationic substances with LC is not compensated by an excess of AGP content and/or that the AGP content in the plasma drops.

Thus, the loading for transfer of the excess of metabolism products increases on the main transport proteins with LC at the compensation and subcompensation stages. This is compensated for, first, by conformational changes of HSA that lead to an increased binding ability of HSA and, second, an enhanced transport function of LP and AGP.

For LC patients in the clinical decompensation stage, the binding ability of HSA to anionic hydrophobic metabolites decreases sharply relative to the compensation, subcompensation, and healthy-donor groups. This is due in all probability to hypoalbuminemia with suppressed liver protein-synthesis function and hyperproduction of anionic hydrophobic metabolites that block the binding sites on albumin. Fluorescence spectra of the NR probe are consistent with an increased contribution of LP and a reduce contribution of HSA to the transport of uncharged hydrophobic metabolites. This can be explained by a progressive infusion into the blood of pathological neutral hydrophobic metabolites and the disruption of the LP/HSA concentration ratio in blood plasma. The binding ability of AGP decreases further relative to that of patients with compensation and subcompensation stages of the disease (Fig. 2). This is apparently due to the increased infusion into blood of cationic hydrophobic substances that block binding sites on AGP and to a reduced concentration of AGP. However, it is noteworthy that the binding ability of AGP in the clinical decompensation stage of LC remains high, like for the healthy donors. 
Thus, as the pathological process deepens and transitions into the decompensation stage of LC, the transport capabilities of HSA and AGP decrease and the binding ability of LP remains high.

Conclusion. The fluorescence probe method using variously charged fluorescent probes (8-anilinonaphthalene1-sulfonate, Quinaldine red, Nile red) can identify changes in the binding of ligands by transport proteins of blood plasma with liver cirrhosis. Development of this disease is accompanied by increased transfer loading of an excess of metabolic products on the main transport proteins that is compensated, first, by conformational changes of HSA and LP that lead to increased binding ability of albumin (according to ANS probe) and, second, by enhancing the transport function of LP (according to NR probe) and AGP (according to QR probe). As the pathological process deepens and transitions to the decompensation stage of LC, the transport capabilities of HSA and AGP decrease and the binding ability of LP remains high. The sequence of changes in binding ability of the main transport proteins of blood plasma with LC, which can be monitored using the fluorescence intensity peak of ANS, QR, and NR probes and the ratio $I_{554} / I_{592}$, which reflects the distribution of NR probe between LP and HSA fractions in whole blood plasma, is established. The relative simplicity of such analysis enables the fluorescence probe method to be proposed for rapid estimation of the disease stage of LC in clinical practice.

Acknowledgments. The work was performed with financial support from the Belarus Republic Foundation for Basic Research (grant F01-169).

\section{REFERENCES}

1. C. Trautwein, K. Boker, and M. P. Manns, Gut, 35, 1163-1166 (1994).

2. P. Tessan, Curr. Opin. Clin. Nutr. Metab. Care, 6, 79-85 (2003).

3. V. G. Kukes, Clinical Pharmacology [in Russian], GEOTAR, Moscow (1999).

4. N. Kh. G. Kholford and L. Z. Benet, Basic and Clinical Pharmacology [in Russian], B. G. Kattsung, ed., BINOM, Moscow (1998), 53-71.

5. M. B. Brown, T. E. Edmonts, J. N. Miller, D. P. Riley, and N. J. Seare, Analyst, 118, 407-410 (1993).

6. D. E. Epps, T. J. Raub, and F. J. Kezdy, Anal. Biochem., 227, 342-350 (1995).

7. S. M. Altamentova, N. Shaklai, R. Arav, and Y. I. Miller, Clin. Chim. Acta, 271, 133-149 (1998).

8. Yu. A. Vladimirov and G. L. Dobretsov, Fluorescent Probes in Biological Membrane Research [in Russian], Nauka, Moscow (1980).

9. D. K. F. Meijer and P. van der Sluijs, Pharm. Res., 6, 105-117 (1989).

10. F. Herve, S. Urien, E. Albengres, and J. C. Duche, Clin. Pharmacokinet., 26, 44-58 (1994).

11. U. Kragh-Hansen, Dan. Med. Bull., 37, 57-84 (1990).

12. J. M. H. Kremer, J. J. Wilting, and L. M. H. Janssen, Pharmacol. Rev., 40, 1-48 (1988).

13. T. Tokui, C. Kuroiwa, S. Muramatsu, Y. Tokui, K. Sasagawa, T. Ikeda, and T. Komai, Biopharm. Drug Dispos., 16, 91-103 (1995).

14. K. M. Wasan and S. M. Cassidy, J. Pharm. Sci., 87, 411-424 (1998).

15. Y. M. Lopuchin, G. E. Dobretsow, and Y. A. Gryzunov, Bull. Exp. Biol. Med., 130, 615-619 (2000).

16. A. I. Ivanov, V. B. Gavrilov, D. A. Furmanchuk, O. V. Aleinikova, S. V. Konev, and G. V. Kaler, Clin. Exp. Med., 2, 147-155 (2002).

17. G. C. Child and J. G. Turcott, in: The Liver and Portal Hypertension, C. G. Child, ed., Saunders, Philadelphia (1964), 50-76.

18. R. N. Pugh, I. M. Murray-Lyon, J. L. Dawson, M. C. Pietroni, and R. William, Br. J. Surg., 80, 646-649 (1973).

19. H. Takikawa, Y. Sekiya, M. Yamanaka, and Y. Sugiyama, Biochim. Biophys. Acta, 1244, 277-282 (1995).

20. P. Greenspan and S. D. Flowler, J. Lipid Res., 26, 781-789 (1985).

21. H. Knobler, M. Fainaru, and D. Sklan, J. Chromatogr., 421, 136-140 (1987).

22. N. L. Vekshin, Biofizika, 41, 1176-1179 (1996).

23. J. B. F. Lloyd, Nature Phys. Sci., 231, 64-65 (1971).

24. H. Imamura, T. Maruyama, and M. Otagiri, Biol. Pharm. Bull., 16, $926-929$ (1993).

25. H. Imamura, T. Maruyama, H. Okabe, H. Shimada, and M. Otagiri, Pharm. Res., 11, 566-570 (1994). 
26. R. Zini, D. Morin, C. Salvadori, and J. P. Millement, Br. J. Clin. Pharm., 29, 9-18 (1990).

27. S. Curry, P. Brick, and N. P. Franks, Biochim. Biophys. Acta, 1441, 131-140 (1999).

28. M. A. Landau, Molecular Nature of Separate Physiological Processes [in Russian], Nauka, Moscow (1985).

29. A. I. Ivanov, E. A. Korolenko, E. V. Korolik, V. V. Sarnaskaya, S. P. Firsov, T. A. Babushkina, R. G. Zhbankov, and V. G. Nikolaev, in: New Developments in Liver Cirrhosis Research, T. M. Chen, ed., Nova Science Publishers Inc., New York (2006), 103-122.

30. S. Sherlock and J. Dooley, Liver Disease and Bile Elimination Pathways [in Russian], GEOTAR-Meditsina, Moscow (1999). 